\title{
Idosas sedentárias apresentam equilíbrio estático e dinâmico prejudicados
}

\author{
Sedentary elderly presents harmed static and dynamic balance
}

PÂmella Mayara Ferreira de MAtos ${ }^{\mathrm{I}}$

Daniela Bassi-Dibai ${ }^{1}$

Thamyres da CruZ Miranda ${ }^{1}$

AdRIANA Sousa RÊGO ${ }^{1}$

Karla Virginia Bezerra de Cas-

TRO SOARES ${ }^{1}$

${ }^{1}$ Universidade Ceuma, São Luís/

MA - Brasil

\begin{abstract}
Resumo A pesquisa teve por objetivo comparar o equilíbrio estático e dinâmico entre idosas ativas e sedentárias. Trata-se de um estudo transversal, composto por dois grupos distintos: Grupo ativo (GA), com 35 idosas praticantes de atividade física, e Grupo sedentário (GS), com 35 idosas sedentárias. Utilizaram-se os seguintes instrumentos: questionário investigativo sobre o histórico de quedas, avaliação estabilométrica, realizada com base na baropodometria na plataforma de pressão, para mensurar o nível de oscilação da amostra e teste Time Up And Go, para a avaliação do risco de queda. Os dados estabilométricos apontaram que, na condição de olhos fechados, o Grupo ativo (GA) apresentou menores oscilações quando comparado ao Grupo sedentário (GS) para as oscilações nos planos anteroposterior $(\mathrm{p}=0,0018)$ e médio- lateral ( $\mathrm{p}$ $=0,007)$. Quanto ao risco de cair, o Teste Timed up and Go - TUG - demonstrou que os idosos ativos realizaram o teste em menor tempo quando comparados aos idosos sedentários com ( $\mathrm{p}=0,0001)$, o que denota menor risco de cair. Dessa forma, as idosas praticantes de atividade física possuem maior estabilidade postural e menor risco de quedas, quando comparadas a idosas sedentárias.
\end{abstract}

Palavras-chave: Equilíbrio. Idosos. Queda.

Aвstract The research aimed to compare the static and dynamic balance between active and sedentary elderly women. This is a cross-sectional study, composed of two distinct groups: Active group (GA), with 35 elderly women practicing physical activity, and Sedentary group (GS), with 35 sedentary elderly women. The following instruments were used: investigative questionnaire on the history of falls, stabilometric evaluation, performed based on baropodometry on the pressure platform, to measure the level of oscillation of the sample and Time Up And Go test, to assess the risk of falling. The stabilometric data showed that, in the closed eyes condition, the active group (GA) showed less oscillations when compared to the sedentary group (GS) for oscillations in the anteroposterior $(\mathrm{p}=0.0018)$ and mid-lateral $(\mathrm{p}=0.007)$ planes. As for the risk of falling, the Timed up and Go Test - TUG - demonstrated that the active older adults performed the test in less time when compared to sedentary older adults with $(\mathrm{p}=0.0001)$, which denotes a lower risk of falling. Thus, elderly women who practice physical activity have greater postural stability and less risk of falls, when compared to sedentary women.

Keywords: Balance. Elderly. Fall 


\section{INTRODUÇÃO}

O traçado demográfico populacional no último século sofreu amplas mudanças em advento de explosão científica e tecnológica, aliada a fatores de ordem econômica, sanitária e social. Essas modificações deram origem a um fenômeno denominado de transição demográfica, influindo diretamente na estrutura etária da população. ${ }^{1,2}$

A ascensão populacional de idosos é uma realidade atual em esfera local, regional e mundial. Segundo menções da Organização Mundial de Saúde (OMS), até 2025 a população mundial de idosos atingirá a margem de 1,2 bilhões de indivíduos. E o Brasil representará um dos seis países com maior número de idosos na escala mundial. ${ }^{3}$

O envelhecimento é um processo fisiológico natural com dinâmica progressiva que gera modificações físico-cognitivas, favorecendo o surgimento de doenças que atribuem inúmeros prejuízos à qualidade de vida do idoso. Suas consequências atingem diretamente a perda da autonomia e independência, tornando o idoso frágil e susceptível a eventos incapacitantes, entre eles as quedas. Portanto, intervenções multidisciplinares voltadas à saúde, ao bem-estar e, principalmente, à qualidade de vida dessa população são imprescindíveis. ${ }^{4,5}$

O evento da queda é compreendido como um deslocamento involuntário do corpo a um nível inferior em relação ao inicial, sem que haja reação em tempo hábil para manutenção postural, com causa e circunstâncias multifatoriais. Atualmente, é visto como uma epidemia no Brasil, tornando-se um dos principais problemas de saúde pública por suas consequências e o alto índice de mortalidade da população idosa. ${ }^{6,7}$

A ocorrência de quedas é variável, pois nem todos envelhecem da mesma forma, podendo haver manifestações mais recentes ou tardias das perdas neurológicas, de mobilidade e do equilíbrio postural. ${ }^{8}$

No que diz respeito ao equilíbrio, alguns autores o definem como a habilidade de controle do centro de gravidade dentro de uma base de suporte e a estabilometria é considerada atualmente, como uma das mais precisas formas de avaliação, permitindo quantificar as oscilações do corpo resultantes da tentativa de manter os segmentos anatômicos alinhados sobre a base de sustentação, ou seja, os pés. ${ }^{9}$

Atualmente, a prática de atividade física tem sido utilizada como importante estratégia para minimizar os efeitos degenerativos do envelhecimento. ${ }^{10}$ Nesse sentido, o presente estudo se justifica, ao avaliar até que ponto a atividade física direcionada a idosos é efetiva na diminuição das oscilações de equilíbrio e manutenção do controle postural. Torna-se primordial reduzir o evento de quedas.

Diante disso, propõe-se a hipótese de que idosas ativas apresentam menor oscilação no equilíbrio e menor risco de quedas do que as idosas sedentárias. Assim, o objetivo deste estudo foi comparar o equilíbrio estático e dinâmico entre idosas ativas e sedentárias.

\section{MATERIAIS E MÉTOdos}

Tratou-se de um estudo analítico de corte transversal, realizado no município de São Luís (MA, Brasil), com 70 mulheres com idade entre 60 e 75 anos, divididas em dois grupos distintos, conforme o nível de atividade física, onde foi utilizado o Ques- 
tionário Internacional de Atividade Física (IPAQ): Grupo de idosas praticantes de atividade física (GA) e Grupo de idosas sedentárias (GS).

O GA $(n=35)$ foi composto por participantes do projeto UNICEUMA Sem Fronteiras (Universidade CEUMA, São Luís) e do Programa de Atenção ao Idoso do Estado do Maranhão (São Luís). As idosas desses programas realizam atividade física similar, de baixo a médio impacto, por no mínimo seis meses, numa frequência de duas vezes semanais, por 50 minutos de duração. As atividades físicas realizadas nas duas instituições eram idênticas e correspondentes ao nível III e IV de aptidão física, ou seja, fisicamente independentes e fisicamente aptos ou ativos. ${ }^{11} \mathrm{O}$ conjunto de atividades era composto por caminhadas e alongamentos para membros superiores e inferiores.

O GS ( $n=35)$ foi formado por frequentadoras da Associação Beneficente de Mães da Vila Nova que não praticam atividade física há no mínimo seis meses.

Foram excluídas do estudo mulheres com qualquer afecção ortopédica que influenciem na deambulação, com ou sem auxílio de dispositivo, e aquelas com distúrbios neurológicos, as que estivessem fazendo uso de medicamentos psicotrópicos ou qualquer fármaco que comprometesse a fidedignidade dos testes, ou ainda as que apresentavam alterações cognitivas.

\section{Procedimentos}

Inicialmente, foi realizada uma reunião de apresentação do estudo e assinatura do termo de consentimento livre e esclarecido. Na coleta de dados, foi empregado um questionário multidimensional elaborado pelo pesquisador com perguntas de caráter sociodemográfico, histórico de saúde atual, histórico de quedas nos últimos 12 meses, utilização de fármacos e a realização de atividade física.

A avaliação do estado cognitivo foi estabelecida por intermédio de um Mini Exame do Estado Mental (MEEM) de Folstein et al. (1975), com o escore mínimo para inclusão de 17. Esse teste é composto por duas fases para investigação das funções cognitivas. A primeira investiga orientação, memória e atenção, totalizando 21 pontos; a segunda verifica a capacidade de nomeação, de obediência a um comando verbal e a um escrito, de redação livre de uma sentença e de cópia de um desenho complexo (polígonos), perfazendo nove pontos. O escore total é de 30 pontos.

O Questionário Internacional de Atividade Física (IPAQ) versão curta foi utilizado para especificar o nível de atividade física das participantes do estudo e classificar os grupos GA e GS. Esse questionário foi validado e testado por Matsudo et al. (2001), e consiste em sete perguntas abertas, onde se estipula o tempo semanal que o indivíduo gasta nas diferentes atividades físicas. O mesmo foi aplicado pelos pesquisadores, a fim de evitar qualquer interpretação errônea pelos participantes.

O Timed Up \& Go (TUG) foi usado para classificar o risco de cair de acordo com o tempo gasto em que o idoso leva para levantar-se de uma cadeira, percorrer uma distância de três metros caminhando, girar $180^{\circ}$, retornar à cadeira e sentar-se. ${ }^{12}$

As oscilações do equilíbrio foram mensuradas por intermédio de análise estática 
no Baropodômetro Digital Footwork ${ }^{\circledast}$ portátil (AM3/IST, Arkipelago, São Paulo, SP) instrumento que consiste em uma plataforma de pressão, de revestimento em policarbonato, com capacitores ou sensores de pressão calibrados, que acoplado a um computador, registra os deslocamentos do centro de pressão (CP) no plano da plataforma $(X, Y)$, nas direções anteroposteriores $(\mathrm{Y})$ e mediolaterais $(\mathrm{X})$, por meio da pressão exercida na plataforma pela planta dos pés. ${ }^{13}$

As idosas foram avaliadas individualmente em dois momentos: primeiro com os olhos abertos e depois de olhos fechados, recebendo a instrução de permanecer imóvel sobre a plataforma, com os pés paralelos em postura relaxada e braços ao longo do corpo. Foi solicitado que durante a avaliação as participantes se mantivessem em silêncio, olhando para um ponto fixo marcado na parede a dois metros de distância da plataforma, na altura dos olhos. ${ }^{14}$

\section{Tamanho da amostra}

O cálculo do tamanho da amostra foi realizado utilizando o software Ene, Versão 3.0 (Universidade Autônoma de Barcelona, Espanha). A variável considerada para o cálculo foi o tempo gasto no TUG, baseado em literatura prévia. ${ }^{15}$ Portanto, usando poder estatístico de $80 \%$ e alfa de $5 \%$, foi estimado um tamanho amostral de 14 pacientes por grupo. Considerando a possível perda amostral, foram inseridas 35 voluntárias.

O presente estudo foi baseado nas normas reguladoras da resolução 466/12 do Conselho Nacional de Saúde que regulamenta as pesquisas envolvendo seres humanos. $\mathrm{O}$ mesmo foi aprovado pelo Comitê de Ética em Pesquisa com Seres Humanos da Universidade CEUMA, sob protocolo número 541.619.

\section{Análise estatística}

Inicialmente, a normalidade dos dados foi verificada pelo teste Kolmogorov-Smirnov. Após confirmada a normalidades dos dados, seguiu-se com Qui-quadrado para as variáveis categóricas e Teste T para amostras independentes, para comparação entre os grupos idosas ativas e idosas sedentárias.

O intervalo de confiança 95\% (IC95\%) das diferenças entre os grupos foi calculado.

Os dados foram analisados usando o software SPSS (versão17.0; Chicago, IL, USA), com nível de significância de 5\%.

\section{RESUlTAdos E discussão}

Foram avaliadas 70 mulheres. A média (DP) de idade para o GA foi de 70,2 $(6,32) \mathrm{e}$ do GS de 68,22 (7,03).

A Tabela 1 apresenta dados referentes à caracterização sociodemográfica da amostra

Cabe aqui ressaltar que, apesar de apresentarem maior média de idade e já estarem aposentadas, as idosas ativas exibiram menor prevalência de quedas, levando a concluir que a prática de uma atividade física regular pode ser efetiva na prevenção de quedas, uma vez que, por possuírem idade maior, teoricamente deveriam mostrar maior susceptibilidade ao evento, em função de sua fragilidade, visto vez que a literatura aponta que quanto maior a idade tanto maiores são as perdas físico-cognitivas. Além disso, o fato de algumas sedentárias ainda estarem ativas e no mercado de trabalho também deveria contribuir para melhor manutenção de suas capacidades funcionais. ${ }^{16}$ 
Tabela 1 - Caracterização das variáveis sociodemográficas obtidas por intermédio de questionário dos grupos GA e GS.

\begin{tabular}{l|c|c|c|c}
\hline \multicolumn{1}{c|}{ Grupo } & GA $(\mathbf{n}=\mathbf{3 5})$ & GS $(\mathbf{n}=\mathbf{3 5})$ & p valor & TOTAL \\
\hline Escolaridade $\mathbf{n}(\%)$ & & & $0,058^{*}$ & \\
\hline Até o $1^{\circ}$ grau completo & $8(22,85)$ & $14(40)$ & & $22(31,42)$ \\
\hline $2^{\circ}$ grau completo & $15(42,85)$ & $17(48,57)$ & & $32(45,72)$ \\
\hline $3^{\circ}$ grau completo & $12(34,28)$ & $4(11,42)$ & & $16(22,86)$ \\
\hline Estado civil $\mathbf{n}$ (\%) & & & 0,76 & \\
\hline Solteira & $2(5,71)$ & $9(25,71)$ & & $11(15,72)$ \\
\hline Casada & $15(42,85)$ & $7(20)$ & & $22(31,42)$ \\
\hline Viúva & $15(42,85)$ & $12(34,29)$ & & $27(38,57)$ \\
\hline Outro & $3(8,59)$ & $7(20)$ & & $10(14,29)$ \\
\hline Profissão $\mathbf{n}$ (\%) & & & $0,048^{*}$ & \\
\hline Ativa & $6(17,14)$ & $15(42,85)$ & & $21(30)$ \\
\hline Aposentada & $29(82,85)$ & $20(57,14)$ & & $49(70)$ \\
\hline Renda Familiar $\mathbf{n}(\%)$ & & & $0,008^{*}$ & \\
\hline Menor ou igual a 1 salário & $6(17,15)$ & $11(31,42)$ & & $17(24,29)$ \\
\hline Entre 2 e 3 salários & $14(40)$ & $17(48,57)$ & & $31(44,28)$ \\
\hline Mais de 3 salários & $15(42,85)$ & $7(20)$ & & $22(31,42)$ \\
\hline Mora sozinha n (\%) & & & 0,66 & \\
\hline Sim & $9(25,71)$ & $5(14,28)$ & & $14(20)$ \\
\hline Não & $26(74,28)$ & $30(85,71)$ & & $56(80)$ \\
\hline Dorme sozinha n $\%)$ & & & 0,27 & \\
\hline Sim & $17(47,57)$ & $12(34,28)$ & & $29(41,42)$ \\
\hline Não & $18(51,43)$ & $23(65,71)$ & & \\
\hline
\end{tabular}

GA: Grupo de idosas ativas, GS: Grupo de idosas Sedentárias, DP - desvio padrão. Teste de Qui-quadrado, $\mathrm{p} \leq 0,05$.

Quando comparado o índice de prevalência de quedas aqui encontrado aos valores do índice médio nacional de 27,6\%, observa-se divergência aos percentuais resultantes da amostra total (31,43\%), e mais ainda aos resultados de prevalência da amostra de idosas sedentárias que foi de $40 \%$. Entretanto, já foram encontrados em estudos recentes índices de até 53,6\%, per- mitindo afirmar que o evento queda tem estado presente na rotina de idosos e que seus índices têm aumentado, apesar das políticas nacionais voltadas ao idoso e ao envelhecimento saudável. ${ }^{17,18}$

Os resultados apresentados na Tabela 2 correspondem à comparação entre grupos para as variáveis de oscilação em Base de sustentação, Amplitude de deslocamento 
do centro de pressão no eixo anteroposterior e Amplitude de deslocamento do centro de pressão no eixo médio-lateral e do TUG, nos grupos de idosas ativas e sedentárias, considerando as condições olhos abertos e olhos fechados.

Tabela 2 - Comparação entre grupos para as variáveis e condições de equilíbrio estático e equilíbrio dinâmico das idosas avaliadas.

\begin{tabular}{l|c|c|c|c|c}
\hline Variáveis/ condições & GA $(\mathrm{n}=35)$ & GS $(\mathrm{n}=35)$ & IC 95\% & $\begin{array}{c}\text { Tamanho } \\
\text { do efeito }\end{array}$ & p valor \\
\hline Base de sustentação $(\mathbf{c m})$ & & & & & \\
\hline BSOA & $14,43(2,62)$ & $14,51(2,83)$ & $-1,37,1,22$ & -0.03 & 0,91 \\
\hline BSOF & $14,54(2,23)$ & $14,58(2,80)$ & $-1,25,1,17$ & -0.02 & 0,94 \\
\hline COPap $(\mathbf{c m})$ & & & & & \\
\hline COPOA & $2,01(0,77)$ & $2,44(1,31)$ & $-0,94,0,08$ & -0.40 & 0,09 \\
\hline COPOF & $2,32(0,79)$ & $3,34(1,56)$ & $-1,61,-0,43$ & -0.82 & $0,001^{*}$ \\
\hline COPml $(\mathbf{c m})$ & & & & & \\
\hline COPOA & $2,16(1,07)$ & $2,94(2,53)$ & $-1,71,0,14$ & -0.40 & 0,09 \\
\hline COPOF & $2,18(1,05)$ & $3,86(2,32)$ & $-2.53,-0,81$ & -0.93 & $0,001^{*}$ \\
\hline Dinâmica & & & & & \\
\hline TUG $(\mathbf{s e g})$. & $9,87(2,06)$ & $12,97(3,74)$ & $-4,54,-1,66$ & -1.03 & $0.001^{*}$ \\
\hline
\end{tabular}

Dados apresentados em média (DP). GA: Grupo de idosas praticantes de atividade física; GS: Grupo de idosas sedentárias; BSOA: Base de Sustentação - Olhos Abertos; BSOF: Base de sustentação - Olhos Fechados; COPap: Amplitude de deslocamento do centro de pressão no eixo anteroposterior; COPml: Amplitude de deslocamento do centro de pressão no eixo médio-lateral; COPOA - Condição olhos abertos; COPOF - Condição olhos fechados; TUG: Time Up \& Go.

${ }^{*}$ Diferenças significativas entre os grupos ativas e sedentárias, $\mathrm{p} \leq 0,05$.

Os achados para a avaliação de equilíbrio postural estático entre os grupos GA e GS na condição olhos abertos não apresentaram diferença significativa para as variáveis ( $p>0,05)$. As médias para o deslocamento da base de sustentação nos grupos exibiram alterações ao serem correlacionadas, principalmente quando submetidos à condição olhos fechados com médias de 14,54 no grupo atividade física e 15,04 para as sedentárias. Entretanto, ainda que essas oscilações tenham sido verificadas ao se avaliar as idosas sedentárias, essa variável não mostrou significância $(\mathrm{p}=0,65)$.
Laughton et al. (2003) encontraram resultados semelhantes ao nosso em seu estudo para os deslocamentos anteroposterior e médio lateral em ambas as condições, porém para a variável base de sustentação os achados divergem, pois, ao comparar idosos ativos e sedentários, constatou maiores deslocamentos da largura base de sustentação nos idosos sedentários com valor de $\mathrm{p}=$ $0,003^{19}$.

Para os resultados das médias entre grupos, quando retirada a informação visual, as sedentárias obtiveram maiores oscilações de $\mathrm{COP}$ anteroposterior e COP médio lateral 
quando comparadas às ativas com $\mathrm{p}=0,0018$ e p $=0,007$ respectivamente. Esses achados encontram justificativa nas afirmativas de Barbosa et al. (2001), ao explicar que, quando o conjunto de informações visuais, vestibulares e proprioceptivas não interage de forma eficaz, ocorre uma instabilidade no estado de equilíbrio corporal, podendo gerar um evento predisponente à quedas.

Em estudo de Bruniera et al. (2014), que teve por objetivo comparar a estabilidade postural de idosos praticantes de uma atividade física e idosos institucionalizados sedentários, encontraram-se resultados semelhantes aos do presente estudo, ao constatar um maior deslocamento na amplitude de COPap, COPml e largura da base de sustentação para situação olhos fechados em idosos sedentários, apontando para um maior risco de cair ${ }^{20}$.

Puzzi et al. (2016) também identificaram, por meio de avaliação estabilométrica, maiores oscilações posturais para idosos sedentários, encontrando diferenças estatisticamente significativas em grande parte das variáveis na postura estática, especialmente no aumento da largura da base de sustentação $(p=0,048)^{8}$. Essa diferença entre $o$ presente estudo e os demais em relação aos resultados e significância da variável base de sustentação pode se justificar pela maior fragilidade de idosos sedentários institucionalizados quando comparados a idosos sedentários não institucionalizados.

Em todas as condições e variáveis o grupo de idosas praticantes de atividade física (GA) obteve médias menores de oscilações que o grupo de sedentárias (GS), revelando maior nível de equilíbrio e, por conseguinte, maior risco de quedas para o grupo de ido- sas sedentárias. Os resultados ora obtidos nos levam a especular que a atividade física possa ter proporcionado maior incremento e manutenção do sistema neuromuscular das idosas ativas.

No que se refere à avaliação dinâmica postural, o teste TUG indicou menor nível de mobilidade funcional, com média 12,97 para as sedentárias que se enquadraram ao nível de risco moderado de quedas, enquanto o grupo ativo, com média 9,87, enquadrou-se no nível de baixo risco de cair, segundo a classificação de Bischoff et al. (2003) e diferença significativa descrita pelo valor de $\mathrm{p}<0,0001^{13}$.

Padoin et al. (2010), ao comparar o risco de cair entre 55 idosas praticantes e não praticantes de atividade física, obtiveram melhor desempenho no teste TUG ( $p=0,001)$ para o grupo de idosas ativas, indicando a atividade física como medida preventiva de quedas $^{21}$. Ribeiro et al. (2013) também verificaram que os idosos ativos realizaram o teste em menor tempo quando comparados aos idosos sedentários, demonstrando diferença significativa entre os grupos $\mathrm{p}=0,0057$.

Mais recentemente, Martins et al. (2016) avaliaram a influência da institucionalização e da prática de atividade física no equilíbrio e na mobilidade funcional de 61 idosos, obtendo significância para TUG ( $p$ $=0,001$ ), no grupo ativo, assim como melhor equilíbrio e mobilidade funcional 8,4 $(2,03)$ segundos para ativas em relação ao grupo sedentário $16,1(12,9)$ segundos ${ }^{22}$, resultados esses que corroboram com os encontrados no presente estudo.

Este estudo possui algumas limitações que devem ser citadas. O nível de atividade física foi avaliado somente por questio- 
nário subjetivo, podendo este subestimar ou superestimar o nível de atividade física do indivíduo. Sabe-se que outros métodos como pedômetros e acelerômetros podem ser utilizados com o objetivo de quantificar de forma mais fidedigna os níveis de atividade física.

\section{CONSIDERAÇõeS FINAIS}

Podemos concluir que idosas sedentárias apresentam maior eventos de queda. Frente ao exposto, a prática de atividades físicas deve ser preconizada nessa população, uma vez que a mesma contribui para a manutenção do equilíbrio postural, reduzindo o risco de quedas.

\section{REFERÊNCIAS}

1. FLORES, L. P. O. O Envelhecimento da população brasileira. Redeca, v. 2, n. 1, jan./jun. 2015, p. 86-100.

2. COSTA, C. K. F. et al. Envelhecimento populacional e a necessidade de reforma da saúde pública e da previdência social brasileiras. A Economia em Revista [S. 1.], v. 19, dez. 2011.

3. IBGE. Instituto Brasileiro de Geografia e Estatística. Síntese de Indicadores Sociais. Uma análise das condições de vida da População Brasileira, 2010. Disponível em: <http://www.ibge.gov. br/home/estatistica/populacao/condicaodevida/indicadoresminimos/sinteseindicsociais2010/ SIS_2010.pdf.> Acesso em: 2 mar. 2017.

4. SCALZO, P.L. et al. Efeito de um treinamento de equilíbrio em um grupo de mulheres idosas da comunidade: estudo piloto de uma abordagem específica, não sistematizada e breve. Acta fisiátrica [S. 1.], v. 14, mar. 2007.

5. CAMPOS, A. C. V.; FERREIRA, E. F.; VARGAS, A. M. D. Determinantes do envelhecimento ativo segundo a qualidade de vida e gênero. Ciência \& Saúde Coletiva, v. 20, n. 7, p. 2.221-2.237, 2015.

6. SCHEICHER, M. E.; ALVES, N. B. Equilíbrio postural e risco para queda em idosos da cidade de Garça, SP. Rev. Bras. Geriatr. Gerontol; 14 (4), 2011, 763-768.

7. PERRACINI, M. R. Prevenção e Manejo de Quedas no Idoso. In: Ramos L.R.; Toniolo Neto J. Geriatria e Gerontologia. Guias de Medicina Ambulatorial e Hospitalar/ Unifesp-Escola Paulista de Medicina. São Paulo: Editora Manole, 2005.

8. PUZZI, P. C. M. Análise baropodométrica do apoio plantar em idosos e sua relação com as quedas. Mestre em Fisioterapia da Senescência. Universidade Fernando Pessoa; 2016. Disponível em: < http://hdl.handle.net/10284/5744> Acesso em: 7 mar. 2017.

9. BANKOFF, A. D. P. et al. Estudo do equilíbrio corporal postural através do sistema de baropodometria eletrônica. Conexões, v. 2, n. 2, 2007.

10. DALLA DÉA, V. H. S. et al. Envelhecimento [recurso eletrônico]: Informações, programa de atividade física e pesquisa, 1. ed. São Paulo: Phorte, 2016. Disponível em: https://books.google. com.br/books?id=hJYUDgAAQBAJ\&printsec=frontcover\&hl=pt BR\&source=gbs_ge_summar y_r\&cad=0\#v=onepage\&q\&f=false $>$ Acesso em: ago. 2017.

11. TRIBESS, S. Prescrição de exercícios físicos para idosos. Rev. Saúde. Com., v. 1, n. 2, 2005. Disponível em: www.uesb.br/revista/rsc/ojs/index.php/rsc/article/view/3. Acesso em: 25 set. 2016.

12. PERRACINI MR. Prevenção e Manejo de Quedas no Idoso. In: Ramos LR, Toniolo Neto J. Geriatria e Gerontologia. Guias de Medicina Ambulatorial e Hospitalar/ Unifesp-Escola Paulista de Medicina. São Paulo: Editora Manole, 2005. 
13. BISCHOFF, H. A.; STÄHELIN, H. B.; MONSCH, A. U.; IVERSEN, M. D.; WEYH, A.; VON DECHEND. M. et al. Identifying a cut-off point for normal mobility: A comparison of the timed 'up and go' test in community-dwelling and institutionalized elderly women. Age Ageing, 2003; 32 (3): 315-20.

14. SOUZA, L. K. et al. Comparação dos níveis de força e equilíbrio entre idosos praticantes de musculação e de hidroginástica. Rev. Bras. Ativ. Fis. Saúde. 2014, 19 (5): 647-648.

15. LOPES et al. Relação da pressão plantar e amplitude de movimento de membros inferiores com o risco de quedas em idosos. Fisioter. Pesqui. São Paulo, v. 23, n. 2, jun. 2016, p. 172-177.

16. SANTOS, A. C. R.; GONÇALES, A. C. M.; RAMOS, H. M.; CARVALHO, E. R. C. Um estudo sobre mulheres idosas no mercado de trabalho, no município de São José dos Campos, SP. Rev. Univasp. São Paulo, v. 22, n. 40, 2016, p. 825.

17. SIQUEIRA, F. V. et al. Prevalência de quedas em idosos no Brasil: uma análise nacional. Cad. Saúde Pública, v. 27, n. 9, p. 1.819- 1.826, set. 2011.

18. SANTOS, R. K. M. et al. Prevalência e fatores associados ao risco de quedas em idosos adscritos a uma Unidade Básica de Saúde do município de Natal, RN, Brasil. Ciênc. \& Saúde Coletiva, v. 20, n. 12, p. 3.753-3.762, 2015.

19. LAUGHTON, C. A.; SLAVIN, M.; KATDARE, K.; NOLAN, L.; BEAN, J. F.; KERRIGAN, D. C. Aging, muscle activity, and balance control: physiologic changes associated with balance impairment. Gait Posture, Philadelphia, v. 18, n. 2, p. 101-108, 2003.

20. BRUNEIRA et al. Comparison of stability in postural older residents in establishment of long stay and practicing of exercise. Rev. Educ. Fís/UEM, v. 25, n. 2, p. 223-230, 2. trim. 2014. Disponível em: http://www.scielo.br/pdf/refuem/v25n2/1983-3083-refuem-25-02-00223.pdf > Acesso em: nov. 2017.

21. ADOIN, P. G.; GONÇALVES, M. P.; COMARU, T.; SILVA, A. M. V. Análise comparativa entre idosos praticantes de exercício físico e sedentários quanto ao risco de quedas. O mundo da Saúde, São Paulo, v. 34, n. 2, p. 158-164, 2010.

22. MARTINS, F. O. et al. Análise comparativa do equilíbrio nos idosos sedentários e idosos praticantes de atividades físicas. Rev Facul Montes Belos (FMB), v. 9, n. 1, 2016, p. (55-173), 2014.

\section{DADOS DOS AUTORES}

\section{Pâmella Mayara Ferreira de Matos}

Discente do curso de Fisioterapia da Universidade Ceuma, São Luís/MA - Brasil. pamellamatos@ hotmail.com

\section{Daniela Bassi-Dibai}

Docente do curso de Fisioterapia e do Programa de Pós-Graduação em Gestão e Serviços de Saúde da Universidade Ceuma, São Luís/MA - Brasil. danielabassifisio@gmail.com

\section{Thamyres da Cruz Miranda}

Discente do curso de Fisioterapia da Universidade Ceuma, São Luís/MA - Brasil. thamyresmiranda2@gmail.com

\section{Adriana Sousa Rêgo}

Docente do curso de Fisioterapia e do Programa de Pós-Graduação em Gestão e Serviços de Saúde da Universidade Ceuma, São Luís/MA - Brasil. adricefs@yahoo.com.br 


\section{Karla Virginia Bezerra de Castro Soares}

Docente e coordenadora do curso de Fisioterapia da Universidade Ceuma, São Luís/MA - Brasil. karla1441@yahoo.com.br

Submetido em: 9-10-2018

Aceito em: $15-8-2020$ 